\title{
COMPETITION AND COOPERATION MODELS FOR DYNAMIC PRICING OF PERISHABLE PRODUCTS IN A TWO-ECHELON SUPPLY CHAIN
}

\author{
Zhenkai Lou ${ }^{1}$, Xuming Lou ${ }^{2}$ And Fujun Hou ${ }^{1, *}$
}

\begin{abstract}
This paper considers a two-level supply chain involving a supplier and a retailer. The retailer sells perishable products to consumers over a finite time horizon, and the demand is driven by a priceand-utility function. First, we study the noncooperative problem, which is formulated by a Stackelberg model. It is shown that the optimal pricing strategy of the retailer is to reduce a constant amount on the price at the beginning of each stage. Second, we examine the cooperative problem, in which the supplier and the retailer jointly price the product. Maximum selling cycle lengths of the two situations are obtained by analyzing the reasonability of the sales price. We demonstrate that the selling cycle length is extended by cooperation. Moreover, we show that they lower the sales price in the cooperative case so as to maximize the total profit. Meanwhile, an allocation method is provided based on the proportion.
\end{abstract}

Mathematics Subject Classification. 90B50, 93A30.

Received June 7, 2020. Accepted September 2, 2020.

\section{INTRODUCTION}

Dynamic pricing of perishable products is a fascinating topic, in which the retailer sells products within a finite time horizon. In recent years, dynamic pricing strategies have been applied successfully in several fields, such as airlines, fashion retailers, hotels, sporting events, fruits sales, and health care. Because the demand rate is price-dependent and time-varying, retailers always price their products dynamically.

Some factors are involved in the dynamic pricing of perishable products. First, the market demand of a certain perishable product is always finite and time-and-price sensitive. Second, because of the variable utility value of perishable products, dynamic pricing strategies are often adopted by sellers to raise the revenues. In addition, the retailer is concerned with not only its sales revenue but also its costs, which are made up of wholesale costs and stock-holding costs. Because consumers do not participate in pricing decisions, we treat them as known information by a demand function.

Recent years, the literature on joint ordering and pricing of perishable products has been growing substantially. In particular, dynamic joint pricing for perishable products has received a lot of attention [11,15, 19,21]. As is known to all, pricing and inventory control have significant impacts on a retailer's profitability. By jointly

Keywords. Perishable products, dynamic pricing, time-and-price sensitive, maximum selling cycle length.

1 School of Management and Economics, Beijing Institute of Technology, Beijing, P.R. China.

2 School of Economics and Management, Xi'an University of Posts and Telecommunications, Xi'an, P.R. China.

*Corresponding author: houf j@bit.edu.cn 
making decisions, the retailer would lower its inventory cost and raise the revenue. For that reason, we pay no attention to the inventory replenishment when the supplier and the retailer jointly price their products.

Generally, the pricing models can be classified into two categories: continuous-time models and discrete-time models. Because the number of times of altering prices cannot be infinite, we consider the sales cycle to be discrete. In addition, we regard the purchase quantity to be a function of the real-time utility value and the current retail price and ignore the individual purchase behavior of consumers. Similar to Taleizadeh et al. [28], we assume that shortage is not permitted. Both the supplier and the retailer aim to maximize their profit. In addition, they can acquire complete and perfect information.

In practice, Supermarkets often dynamically price perishable items like dairy products. Usually, they will not be on sale when their selling periods last for some time, even they are still within the shelf life. Study on how to determine the optimal selling cycle length of a perishable item is of practical significance. This paper not only discusses the optimal selling cycle length of the non-cooperation model based on the utility of the product, but also analyzes the change on the optimal selling cycle length when the supplier and the retailer jointly make decisions. The aim is to show that the selling cycle length of a perishable product can be extended by centralized decision-making. In addition, sales prices and total profits of the non-cooperation case and the cooperation case are compared.

The remainder of this paper is organized as follows. In Section 2, we briefly review the related literature about dynamic pricing of perishable products. Section 3 describes notations and assumptions, and then presents models for the supplier and the retailer. The noncooperative issue is discussed in Section 4. In Section 5, we focus on analyzing the impact incurred by cooperation, and propose a suitable method to allocate the surplus value. A numerical illustration is given in Section 6. Section 7 summarizes the paper.

\section{Related Literature}

Pricing decisions play a crucial role in managing demand in many industries [3]. As is well known, consumer demand depends on the sale price. Hence, it is imperative for a retailer to trade off the ordering cost, the stockholding cost, and the lost sales cost while making his ordering and pricing decisions [7]. This paper considers one-time ordering issues, and the ordering cost is regarded as a constant that has no impact on the decision making.

The concept of perishable product was first proposed to describe time-sensitive goods [29]. In order to maximize their revenue, many industries, retailers, and service providers raised their revenue through dynamic pricing when facing the problem of selling perishable products $[5,12,13]$. Because of the practicability of dynamic pricing, a significant amount of work has been conducted.

For perishable products, quality always plays an important role $[9,10,21]$. In addition, the utility of a perishable product always varies along with time, and the purchase quantity of consumers depends on both the utility and the sales price [20], which we also adopt. Because of the variable quality (or utility value) of perishable products, dynamic pricing strategies are often used to raise the revenues of sellers. Gallego et al. [12] studied dynamic pricing in an oligopolistic market with a mix of substitutable and complementary perishable assets. Schlosser et al. [27] pointed out that frequent price adjustments are crucial for perishable products. Lu et al. [23] constructed dynamic pricing models by considering replenishment cycle length, replenishment quantity and preservation technology investment.

Some literature focused on consumers' strategic purchase behavior when dealing with ordering and pricing issues, which is mainly involved with competition. Besanko et al. [2] studied an optimal pricing strategy with a retailer and a number of consumers by considering the consumers' strategic behavior on different sales prices. Levin et al. [18] and Papanastasiou et al. [25] separately formulated dynamic-pricing models for a monopolist in the presence of strategic consumers. In addition, the competition between two monopolistic retailers while taking strategic consumers into account was discussed and game-theoretic techniques were applied to obtain equilibrium solutions $[17,24]$. 
Recent years, jointly ordering and pricing issues of suppliers and retailers have attracted many attentions. Generally, if suppliers and retailers jointly make decisions by sharing demand information and revenues, less inventory costs come about [14]. Akcay et al. [1] conducted pioneering work for joint dynamic pricing of perishable products, developed an integrative model of consumer choice based on linear random consumer utilities. Furthermore, Chen et al. [8] proposed methods to obtain an optimal joint pricing policy while considering linear ordering cost, inventory holding, and lost-sales penalty cost. Chen [6] constructed a centralized decision model in the presence of a vendor managed inventory, and discussed the optimal strategies to enhance the revenue of the retailer.

However, despite the plenty of literature with regard to pricing for perishable products, few considers competition and cooperation simultaneously and analyzes the impact incurred by cooperation, which motivates our study. Similar to Liu et al. [20] and Levin et al. [17], we present discrete models instead of continuous ones, by considering the finitude of the number of times of altering prices under complete and perfect information. A novel demand function consisting of the real-time utility value, the real-time sales price and the time-point is proposed. Following our recent research [22], a non-cooperation model and a cooperation model both involving a supplier and a retailer are constructed so as to analyze the differences on sales prices and ordering quantities. Moreover, selling cycle lengths between the two situations are compared. In the first model, the supplier determines a wholesale price, while the retailer determines a wholesale volume and a sales price. In the second model, the supplier and the retailer jointly price the item on the premise of sharing stock-holding cost. An allocation approach is given in the cooperation case to distribute the total profit.

\section{MOdEL FORMUlation}

This section describes the fundamental elements of our models. In addition, all of the assumptions are given and the objective functions of the supplier and the retailer are presented.

\subsection{Notations and assumptions}

We consider a sales scenario composed of a supplier selling a perishable product to end consumers through a retailer. We assume that the product perishes at a certain known time, and therefore the retailer focuses on enhancing its profit and ignores the surplus value of the product when finishing a sales cycle. The supplier has only one opportunity to sell its products. By comparison, the retailer prices and sells its items at multiple stages.

Following many existing papers, in the following discussion, we employ a linear function to depict the utility value of the product for consumers at a certain time. We assume that the potential market demand of the product increases linearly along with the sales time. Furthermore, we believe that the purchase quantity of consumers may not always coincide with their demands, because they also depend on the purchase time and the current retail price. The following notations are used throughout this paper (Tab. 1).

In the above notations, $\alpha, \beta, u^{0}$ and $h$ are constant parameters, which are mainly acquired by historical data and the number of consumers, similar to the viewpoint of Boer et al. [4].

We assume that $\beta$ is higher than $h$. Otherwise, the stock-holding cost of one product through the whole sales cycle would be equal to or higher than its maximum utility value, which usually cannot happen.

Some explanations are made for the rationality and the practicality of the proposed purchase quantity function. Firstly, consumers are always not willing to buy a perishable product when the utility value is lower than the sales price. Secondly, by the decreasing utility function $u(t)$, we know that there is no demand after $t=u^{0} / \beta$. Denoting by $T^{0}=u^{0} / \beta$, it is clear that $T \leq T^{0}$. In the following exploration, we will acquire the maximum selling cycle length, which is assumed to be $T$. Thirdly, the utility value is assumed to be a linear decreasing function of time, for describing a product with a stable change rate of quality. In practice, other types of demand functions are adopted for products with different change rates of quality (e.g., [30]). Finally, similar to the handling of many literature (e.g., [16]), we adopt a linear function of $q(t)$ to describe $Q(t)$ by taking the utility value into consideration. 
TABLE 1. Model parameters.

\begin{tabular}{ll}
\hline \hline Parameters & Definition \\
\hline$T$ & The selling cycle length determined by the retailer \\
$v$ & The wholesale volume determined by the retailer \\
$p$ & The wholesale price determined by the supplier \\
$\alpha$ & The potential demand amount per unit time \\
$K(t)$ & The market capacity within a period of time $t, K(t)=\alpha t$ \\
$\beta$ & The decline rate of utility value of the product for consumers \\
$u^{0}$ & The utility value for consumers at the beginning of the sales \\
$u(t)$ & The utility value of the product for consumers at time $t, u(t)=u^{0}-\beta t$ \\
$q(t)$ & The retail price at time $t$ \\
$Q(t)$ & The purchase quantity per unit time, $Q(t)=\alpha(u(t)-q(t)) / u^{0}$ \\
$h$ & The stock-holding cost per item per unit time \\
$R$ & The rounding function for determining the selling cycle length under \\
$W$ & the discrete scenario \\
$Z$ & The profit of the supplier \\
\hline
\end{tabular}

Moreover, we assume that the wholesale volume of the retailer cannot be less than the purchase amount of the consumers, which suggested that we pay no attention to stock-out issues in the following discussion. Besides, over ordering is unnecessary. Hence, we have

$$
v=\int_{0}^{T} Q(t) \mathrm{d} t
$$

\subsection{Model description}

We next describe the objective models of the supplier and the retailer.

The model of the supplier with $p$ as the unique decision variable can be formulated as follows

$$
\max W=p v,
$$

where $p$ is the decision variable of the supplier, and $v$ is determined by the retailer.

The model of the retailer with $v$ and $q(t)$ as its decision variables is given as follows

$$
\begin{aligned}
& \max Z=\int_{0}^{T}(q(t)-p) Q(t) \mathrm{d} t-h \int_{0}^{T}\left(v-\int_{0}^{t} Q(s) \mathrm{d} s\right) \mathrm{d} t \\
& \text { subject to } \int_{0}^{t} Q(s) \mathrm{d} s \leq K(t), \quad \forall t \in[0, T], \\
& v \leq K(T), \quad \forall t \in[0, T] . \\
& q(t) \leq u(t), \quad \forall t
\end{aligned}
$$

In model (3.2), $v$ and $Q(t)$ are intermediate variables, which are determined by sales prices $q(t)$ of all stages. In practice, the constraints of model (3.2) make sense.

Given a time series: $0=t_{1}<\cdots<t_{n}<t_{n+1}=T$, we discretize the times into $n$ stages. Each stage is denoted by $\left[t_{i}, t_{i+1}\right]$, where $i \in[1, \ldots, n]$. At the beginning of each stage, the retailer declares its sales price. Furthermore, following Liu et al. [20], we assume that the length of each stage $l=1$. Thus, $T=n$ and $t_{i}=i-1$, where the unit can be day, week, etc. Clearly, $T$ (or $n$ ) is also a decision variable of the retailer. Later we will show that all the other decision variables depend on $T$. Thus, $T$ is regarded as an undetermined parameter 
during the acquisition of other decision variables. Finally, we will deal with the maximum selling cycle length $T$.

Then we transform model (3.2) into the following form

$$
\begin{aligned}
\max & Z=\sum_{i=1}^{n} Q\left(t_{i}\right)\left(q\left(t_{i}\right)-p\right)-\sum_{i=1}^{n} h\left(v-\sum_{j=1}^{i} Q\left(t_{j}\right)\right) \\
\text { subject to } & \sum_{i=1}^{m} Q\left(t_{i}\right) \leq K\left(t_{m+1}\right), \quad m \in\{1, \ldots, n\}, \\
& v \leq K\left(t_{n+1}\right), \\
& q\left(t_{i}\right) \leq u\left(t_{i}\right), \quad i \in\{1, \ldots, n\} .
\end{aligned}
$$

In model (3.3), we assume that $u(t), q(t)$ and $Q(t)$ take their values at the beginning of each stage, whereas $K(t)$ takes its value at the end of each stage. Besides, it is assumed that products sold in a certain stage would also incur stock-holding cost as a result of the fact that, they generally cannot be sold just at the beginning of this stage.

\section{DyNAMiC PRICING IN A NON-COOPERATIVE MODEL}

In practice, for some reasons, suppliers and retailers cannot cooperate closely. For example, agreements on cost sharing and revenue allocation may not be able to be reached between them. For this situation, we focus on addressing this dynamic pricing problem in which the supplier and the retailer make separate decisions. The decision order is that the supplier declares its wholesale price first, and then the retailer determines its sales price. In fact, this is a Stackelberg games framework.

We now deal with the Stackelberg game model consisting of (3.1) and (3.3). The backward induction approach is adopted to handle this model, by solving (3.3) to obtain the expression of each $q(t)$.

Because it is difficult to solve model (3.3) directly, we transform its objective function to the following form:

$$
\begin{aligned}
Z & =\sum_{i=1}^{n} Q\left(t_{i}\right)\left(q\left(t_{i}\right)-p\right)-\sum_{i=1}^{n} h\left(v-\sum_{j=1}^{i} Q\left(t_{j}\right)\right) \\
& =\sum_{i=1}^{n} Q\left(t_{i}\right)\left(q\left(t_{i}\right)-p\right)+\sum_{i=1}^{n} \sum_{j=1}^{i} h Q\left(t_{j}\right)-\sum_{i=1}^{n} h v
\end{aligned}
$$

where $q\left(t_{i}\right)$ and $v$ are variables of $Z$ and $p$ is the variable of $W$. Because

$$
\begin{aligned}
\sum_{i=1}^{n} \sum_{j=1}^{i} h Q\left(t_{j}\right) & =h Q\left(t_{1}\right)+\left[h Q\left(t_{1}\right)+h Q\left(t_{2}\right)\right]+\cdots+\left[h Q\left(t_{1}\right)+\cdots+h Q\left(t_{n}\right)\right] \\
& =n h Q\left(t_{1}\right)+(n-1) h Q\left(t_{2}\right)+\cdots+h Q\left(t_{n}\right) \\
& =\sum_{i=1}^{n}\left[(n+1-i) h Q\left(t_{i}\right)\right]
\end{aligned}
$$

$Z$ can be transformed into

$$
Z=\sum_{i=1}^{n}\left[Q\left(t_{i}\right)\left(q\left(t_{i}\right)-p\right)+(n+1-i) h Q\left(t_{i}\right)\right]-\sum_{i=1}^{n} h v .
$$


In the discrete situation, $v$ can be described as follows

$$
v=\sum_{i=1}^{n} Q\left(t_{i}\right)=\frac{\alpha}{u^{0}} \sum_{i=1}^{n}\left(u^{0}-\beta t_{i}-q\left(t_{i}\right)\right) .
$$

For a certain $v$ determined by formula (4.2), we transform $\sum_{i=1}^{n} h v$ into the following expression

$$
\sum_{i=1}^{n} h v=\frac{\alpha}{u^{0}} \sum_{i=1}^{n} n h\left(u^{0}-\beta t_{i}-q\left(t_{i}\right)\right) .
$$

Function (4.1) then can be transformed by using expression (4.3):

$$
Z=\frac{\alpha}{u^{0}} \sum_{i=1}^{n}\left(q\left(t_{i}\right)-p+(1-i) h\right)\left(u^{0}-\beta t_{i}-q\left(t_{i}\right)\right) .
$$

Given a certain $p$, for any $i \in\{1, \ldots, n\}$ we know that

$$
\left(q\left(t_{i}\right)-p+(1-i) h\right)\left(u^{0}-\beta t_{i}-q\left(t_{i}\right)\right)
$$

is a function with a unique variable $q\left(t_{i}\right)$. Hence, we can solve model (3.3) sequentially stage by stage.

We next consider (4.5). Clearly, it is a quadratic function with a negative second-order coefficient. Therefore, we acquired its maximum value when $q\left(t_{i}\right)$ satisfies the following:

$$
q^{*}\left(t_{i}\right)=\frac{u^{0}-\beta t_{i}+p+(i-1) h}{2} .
$$

Meanwhile, the corresponding purchase amount is obtained as follows:

$$
Q^{*}\left(t_{i}\right)=\frac{\alpha}{2 u^{0}}\left[u^{0}-\beta t_{i}-p-(i-1) h\right] .
$$

Given the above results, we obtain the solution of $v$, which guarantees that $Z$ reaches the maximum value, as follows:

$$
v^{*}=\sum_{i=1}^{n} Q^{*}\left(t_{i}\right)=\sum_{i=1}^{n} \frac{\alpha}{2 u^{0}}\left[u^{0}-\beta t_{i}-p-(i-1) h\right] .
$$

Formula (4.8), meanwhile, is the Lagrange multiplier that we are searching for, which is actually a relational expression of $p$ and $v$. Because both the supplier and the retailer acquire complete and perfect information, we transform model (3.1) into the following function by taking (4.8) into account

$$
\max W=\frac{\alpha}{2 u^{0}} \sum_{i=1}^{n}\left[-p^{2}+u^{0} p-\beta t_{i} p-(i-1) h p\right] .
$$

Apparently, (4.9) is also a quadratic function with a negative second-order coefficient, which can be transformed into the following form by considering $n=T$ and $t_{i}=i-1$ :

$$
\begin{aligned}
W & =\frac{\alpha}{2 u^{0}} \sum_{i=1}^{n}\left[-p^{2}+u^{0} p-\beta t_{i} p-(i-1) h p\right] \\
& =\frac{\alpha}{2 u^{0}} \sum_{i=1}^{n}\left[-p^{2}+u^{0} p-(i-1) \beta p-(i-1) h p\right] \\
& =\frac{\alpha}{2 u^{0}}\left[-n p^{2}+\left(n u^{0}-\frac{n(n-1)}{2} \beta-\frac{n(n-1)}{2} h\right) p\right] .
\end{aligned}
$$


We obtain the optimal wholesale price $p$ by solving $\max W$ :

$$
p^{*}=\frac{2 u^{0}-(n-1) \beta-(n-1) h}{4} .
$$

As it is shown, the wholesale price of the supplier depends on the selling cycle length of the product, which implies that the supplier cannot make decisions before the retailer declares its selling cycle length. Hence, the selling cycle length is the first one that should be figured out.

After acquiring the value of $p^{*}$, we turn back to formula (4.6) and formula (4.8). By substituting (4.11) into (4.6), the values of all $q^{*}\left(t_{i}\right)$ are obtained as follows:

$$
q^{*}\left(t_{i}\right)=\frac{3}{4} u^{0}-\frac{\beta(i-1)}{2}-\frac{\beta(n-1)}{8}+\frac{(i-1) h}{2}-\frac{(n-1) h}{8} .
$$

And $v^{*}$ is acquired by substituting (4.11) into (4.8):

$$
v^{*}=\frac{n \alpha}{4}-\frac{n(n-1) \alpha \beta}{8 u^{0}}-\frac{n(n-1) \alpha h}{8 u^{0}} .
$$

Also, the wholesale volume is a function of the selling cycle length.

The above solutions are obtained without considering the constraints of model (3.3). Besides, $n$ (or $T$ ) is undetermined. Before determining $n$, we first present the following conclusion:

Proposition 4.1. The optimal pricing strategy of the retailer is to reduce a constant amount on the price at the beginning of each stage.

Proof. Formula (4.6) gives the optimal price of each stage for the retailer. Given the following functions

$$
q^{*}\left(t_{i}\right)=\frac{u^{0}-\beta t_{i}+p+(i-1) h}{2}, q^{*}\left(t_{i+1}\right)=\frac{u^{0}-\beta t_{i+1}+p+i h}{2},
$$

and relations $h<\beta$ and $t_{i+1}-t_{i}=1$, we have

$$
q^{*}\left(t_{i}\right)-q^{*}\left(t_{i+1}\right)=\frac{1}{2}\left(-\beta t_{i}+\beta t_{i+1}-h\right)=\frac{1}{2}(\beta-h)>0 .
$$

Hence, the retailer lowers the price at the beginning of each stage to obtain the maximum profit. Moreover, it is shown that the extent of the reduction with respect to the sales price of any two adjacent stages is a constant.

This proposition actually reveals the regularity of the optimal pricing strategy of each stage. In practice, a constant is convenient for strategy control.

Next, we look for the maximum selling cycle length by examining the constraints.

Clearly, solutions determined by formula (4.11) and formula (4.12) are the unique optimal strategies of the supplier and the retailer, respectively. Hence, they should meet the constraints of model (3.3) so as to guarantee the sales occurs through the whole period.

Firstly, we construct a function $f(n)=u\left(t_{i}\right)-q^{*}\left(t_{i}\right)$ to determine the maximum number of sales periods. It is obtained that

$$
\begin{aligned}
f(n) & =u\left(t_{i}\right)-q^{*}\left(t_{i}\right) \\
& =u^{0}-\beta(i-1)-\left[\frac{3}{4} u^{0}-\frac{\beta(i-1)}{2}-\frac{\beta(n-1)}{8}+\frac{(i-1) h}{2}-\frac{(n-1) h}{8}\right] \\
& =\frac{1}{4} u^{0}-\frac{\beta(i-1)}{2}+\frac{\beta(n-1)}{8}-\frac{(i-1) h}{2}+\frac{(n-1) h}{8},
\end{aligned}
$$


where $i \in\{1, \ldots, n\}$. In practice, consumers will always not purchase a perishable item when its utility value is lower than its sales price. Hence, $q^{*}\left(t_{i}\right) \leq u\left(t_{i}\right)$ is the necessary condition to ensure that the sales activity occurs at stage $t_{i}$. We show the following inequality to guarantee that the sales activity happens through the whole period:

$$
\frac{1}{4} u^{0}-\frac{\beta(n-1)}{2}+\frac{\beta(n-1)}{8}-\frac{(n-1) h}{2}+\frac{(n-1) h}{8}=\frac{1}{4} u^{0}-\frac{3 \beta(n-1)}{8}-\frac{3(n-1) h}{8} \geq 0 .
$$

Then $n^{*}$ is acquired by rounding function $R\langle\rangle$ :

$$
n^{*}=R\left\langle\frac{1}{4} u^{0}-\frac{3 \beta(n-1)}{8}-\frac{3(n-1) h}{8} \geq 0\right\rangle .
$$

Secondly, for any $m \in\left\{1, \ldots, n^{*}\right\}$,

$$
\sum_{i=1}^{m} Q\left(t_{i}\right)=\frac{m \alpha}{4}-\frac{m(m-1) \alpha \beta}{8 u^{0}}-\frac{m(m-1) \alpha h}{8 u^{0}}<m \alpha .
$$

Given the above, $n^{*}$ determined by formula (4.14) meets the constraints of model (3.3).

Because $T=n^{*}$, the above discussion actually presents the maximum selling cycle length. In others words, the retailer will not gain profit when exceeding $T$.

In addition, for any $m \in\left\{1, \ldots, n^{*}\right\}$, we have

$$
\begin{aligned}
q^{*}\left(t_{i}\right)-p^{*} & =\frac{1}{4} u^{0}-\frac{\beta(i-1)}{2}+\frac{\beta(n-1)}{8}+\frac{(i-1) h}{2}+\frac{(n-1) h}{8} \\
& >\frac{1}{4} u^{0}-\frac{\beta(i-1)}{2}+\frac{\beta(n-1)}{8}-\frac{(i-1) h}{2}+\frac{(n-1) h}{8} \\
& \geq 0,
\end{aligned}
$$

which suggests that the retailer will not sell the product under a price that is lower than $p^{*}$ at any stage.

\section{Dynamic PRICING in A COOPERATIVE MOdel}

Sometimes, suppliers and retailers may jointly make decisions by reaching agreements on costs sharing and profit allocation. In this scenario, the wholesale price does not make any impact on optimal decision [26]. Hence, in the cooperation model discussed in this section, we focus on sales prices of each stage, the ordering quantity, the optimal selling cycle length, and the increased profit rather than the wholesale price.

It is well known that the total profit gained by cooperation is always greater than or equal to the sum of the profit gained by the two participants in the noncooperation situation. Next, we examine the increased value incurred by cooperation when selling perishable products.

Proposition 5.1. The increased value brought by the cooperation between the supplier and the retailer is definitely positive.

Proof. We follow the previous assumption and further assume that the supplier and the retailer share the stock-holding cost of the retail products on the premise of cooperation:

$$
\begin{aligned}
W+\mathrm{Z} & =\sum_{i=1}^{n}\left[Q\left(t_{i}\right) q\left(t_{i}\right)+(n+1-i) h Q\left(t_{i}\right)\right]-\sum_{i=1}^{n} h v \\
& =\frac{\alpha}{u^{0}} \sum_{i=1}^{n}\left[q\left(t_{i}\right)+(1-i) h\right]\left[u^{0}-\beta(i-1)-q\left(t_{i}\right)\right] .
\end{aligned}
$$


By solving $\max (W+Z)$, we obtain the value of $q^{\prime}\left(t_{i}\right)$ at stage $t_{i}$ as follows:

$$
q^{\prime}\left(t_{i}\right)=\frac{u^{0}-\beta(i-1)+(i-1) h}{2} .
$$

By comparing (4.12) and (5.2), we know that $q^{\prime}\left(t_{i}\right) \neq q^{*}\left(t_{i}\right)$ for any $i \in\{1, \ldots, n\}$. Besides, for a quadratic function with a negative second-order coefficient, we know that it has only a unique maximum value.

Thus, we obtain the following proposition:

$$
W^{*}+Z^{*}<\max _{q^{\prime}\left(t_{i}\right)}(W+Z) .
$$

Furthermore, the increased value gained by cooperation is given as follows:

$$
S=\max _{q^{\prime}\left(t_{i}\right)}(W+Z)-W^{*}-Z^{*} .
$$

The above result reveals that the two participants definitely gain more profits by cooperation. In theory, this is because the sales price of each stage has changed, which is also the extreme point of the total profit function.

The increase of the total profit is a necessary condition for the cooperation between the supplier and the retailer. Without this condition, the supplier and the retailer cannot enhance their revenue simultaneously by cooperation.

Besides, the sales quantity $v^{\prime}$ is obtained as follows:

$$
v^{\prime}=\sum_{i=1}^{n} Q^{\prime}\left(t_{i}\right)=\sum_{i=1}^{n} \frac{\alpha}{2 u^{0}}\left[u^{0}+\beta(i-1)-(i-1) h\right]=\frac{n \alpha}{2}+\frac{n(n-1) \alpha \beta}{4 u^{0}}-\frac{n(n-1) \alpha h}{4 u^{0}} .
$$

Because $h<\beta$, we have

$$
\begin{aligned}
q^{*}\left(t_{i}\right)-q^{\prime}\left(t_{i}\right) & =\frac{3}{4} u^{0}-\frac{\beta(i-1)}{2}-\frac{\beta(n-1)}{8}+\frac{(i-1) h}{2}-\frac{(n-1) h}{8}-\left[\frac{u^{0}}{2}-\frac{\beta(i-1)}{2}+\frac{(i-1) h}{2}\right] \\
& =\frac{1}{4} u^{0}-\frac{\beta(n-1)}{8}-\frac{(n-1) h}{8} \\
& >\frac{1}{4} u^{0}-\frac{\beta(n-1)}{4} \\
& >0
\end{aligned}
$$

and

$$
\begin{aligned}
v^{\prime}-v^{*} & =\frac{n \alpha}{2}+\frac{n(n-1) \alpha \beta}{4 u^{0}}-\frac{n(n-1) \alpha h}{4 u^{0}}-\left[\frac{n \alpha}{4}-\frac{n(n-1) \alpha \beta}{8 u^{0}}-\frac{n(n-1) \alpha h}{8 u^{0}}\right] \\
& =\frac{n \alpha}{4}+\frac{3 n(n-1) \alpha \beta}{8 u^{0}}-\frac{n(n-1) \alpha h}{8 u^{0}} \\
& >\frac{n \alpha}{4}+\frac{n(n-1) \alpha h}{4 u^{0}},
\end{aligned}
$$

which implies that in the cooperative situation, the supplier and the retailer will lower the sales price for the sake of a great enhance on the sales quantity. 
Moreover, the optimal price determined by formula (5.2) also needs to meet the constraints of model (3.3). We define a function $g(n)=u\left(t_{i}\right)-q^{\prime}\left(t_{i}\right)$ to determine the maximum number of sales periods. Thus, we have

$$
\begin{aligned}
g(n) & =u^{0}\left(t_{i}\right)-q^{\prime}\left(t_{i}\right) \\
& =u^{0}-\beta(i-1)-\left[\frac{u^{0}}{2}-\frac{\beta(i-1)}{2}+\frac{(i-1) h}{2}\right] \\
& =\frac{u^{0}}{2}-\frac{\beta(i-1)}{2}-\frac{(i-1) h}{2} .
\end{aligned}
$$

The following inequality guarantees that the sales activity happens through the whole period:

$$
\frac{u^{0}}{2}-\frac{\beta(n-1)}{2}-\frac{(n-1) h}{2} \geq 0
$$

Then $n^{\prime}$ is acquired by rounding function $R\langle\rangle$ :

$$
n^{\prime}=R\left\langle\frac{u^{0}}{2}-\frac{\beta(n-1)}{2}-\frac{(n-1) h}{2} \geq 0\right\rangle .
$$

Because

$$
\begin{aligned}
\frac{u^{0}}{2}-\frac{\beta(n-1)}{2}-\frac{(n-1) h}{2}-\left[\frac{u^{0}}{4}-\frac{3 \beta(n-1)}{8}-\frac{3(n-1) h}{8}\right] & =\frac{u^{0}}{4}-\frac{\beta(n-1)}{8}-\frac{(n-1) h}{8} \\
& >\frac{u^{0}}{4}-\frac{\beta(n-1)}{4} \\
& >0,
\end{aligned}
$$

the sales period always longer in the cooperation case than the one in the non-cooperation case. In addition, $u^{0}(m)=u^{0}-\beta m \geq 0$ holds for any $m \in\left\{1, \ldots, n^{\prime}\right\}$. Hence, we have

$$
\sum_{i=1}^{m} Q\left(t_{i}\right)=\frac{m \alpha}{2}+\frac{m(m-1) \alpha \beta}{4 u^{0}}-\frac{n(m-1) \alpha h}{4 u^{0}}<\frac{m \alpha}{2}+\frac{m(m-1) \alpha \beta}{4 u^{0}}<\frac{m \alpha}{2}+\frac{(m-1) \alpha}{4}<m \alpha,
$$

i.e., $q^{\prime}\left(t_{i}\right)$ determined by formula (5.2) meets the corresponding constraints.

The above result shows that the selling cycle length can be extended by lowering the sales price of each stage, which is of practical significance. Apparently, this happens only when the supplier and the retailer jointly make decisions. Moreover, by (4.14) and (5.5), it is shown that the optimal selling cycle length has nothing to do with the potential market demand.

Finally, we propose a solution to allocate the increased profit. In the cooperative game theory, the method of distributing value equally is widely adopted. This approach, however, doesn't take the profit gap between the participants into account. According to the previous profit of each participant, we propose an allocation method based on the proportion.

We denote by $W^{\prime}$ the adjusted profit of the supplier and $Z^{\prime}$ the adjusted profit of the retailer. Then, the formula is given as follows:

$$
W^{\prime}=W^{*}+\frac{W^{*} S}{W^{*}+Z^{*}}, Z^{\prime}=Z^{*}+\frac{Z^{*} S}{W^{*}+Z^{*}} .
$$

Clearly, the allocation determined by formula (5.6) increases the revenue of both the supplier and the retailer. 


\section{A NUMERICAL ILLUSTRATiON}

In this section, we present a general example that meets the previous conditions to visualize the proposed model and conclusions, e.g., the discussed object can be a two-layer supply chain that sells a kind of dairy product with about the shelf life of ten days. Given the potential demand amount per day $\alpha=50$, the decline rate of utility value $\beta=3$, the utility value of the product at the beginning of the sales $u^{0}=32$, and the stock-holding cost per item per day $h=1$. The retailer prices its product at the beginning of each day.

First, we consider the noncooperative situation. Different from the theoretical analysis, the selling cycle length should be determined first. By function (4.14), we obtain the optimal selling cycle length $n^{*}=6$.

According to formula (4.4), the objective function of the retailer is acquired as follows:

$$
Z=\frac{50}{32} \sum_{i=1}^{6}\left[q\left(t_{i}\right)-p+(1-i)\right]\left[32-3(i-1)-q\left(t_{i}\right)\right] .
$$

Then we acquire the expression of the price of each stage

$$
q^{*}\left(t_{i}\right)=\frac{32-2(i-1)+p}{2} .
$$

By formulae (4.7) to (4.9), we obtain the following:

$$
W=p v^{*}=\frac{25}{32} \sum_{i=1}^{6}\left[-p^{2}+32 p-4(i-1) p\right] .
$$

By solving the above quadratic function, we obtain $p^{*}=11$. Through formula (4.7), we know that $Q^{*}\left(t_{i}\right)>0$ for any $i \in\{1, \ldots, 6\}$.

Substituting $p^{*}$ into formula (4.6) and formula (4.8), we further obtain the optimal price of each stage $q^{*}\left(t_{i}\right)=22.5-i$, and the optimal wholesale volume $v^{*} \approx 51.6$. Immediately, the maximum revenues of the supplier and the retailer are $W^{*} \approx 567.2$ and $Z^{*} \approx 393$.

The following graph (Fig. 1) drawn by MATLAB shows variation trend of the optimal price $q^{*}\left(t_{i}\right)$, in which the horizontal axis represents the sales price of each stage, and the vertical axis represents the profit of each stage.

Next, we analyze the case in which the supplier and the retailer jointly price the product to enhance their revenues. By formula (5.5), the maximum number of sales periods $n^{\prime}=9$.

By formula (5.1), we have

$$
W+\mathrm{Z}=\frac{50}{32} \sum_{i=1}^{9}\left(q\left(t_{i}\right)+1-i\right)\left(35-3 i-q\left(t_{i}\right)\right) .
$$

Solving this function, we obtain the solution $q^{\prime}\left(t_{i}\right)=17-i$. Then we get

$$
\max _{q^{\prime}\left(t_{i}\right)}(W+Z)=\frac{50}{32} \sum_{i=1}^{9}(18-2 i)^{2}=1275 .
$$

The optimal supply quantity is obtained by formula (5.4): $v^{\prime} \approx 281.3$.

We obtain the surplus value by formula (5.4), as follows:

$$
S=\max _{q^{\prime}\left(t_{i}\right)}(W+Z)-W^{*}-Z^{*} \approx 314.8 .
$$

The adjusted profits for the two participants are acquired by formula (5.5): $W^{\prime} \approx 753.2, Z^{\prime} \approx 521.8$. Apparently, both the supplier and the retailer significantly enhance their profit by jointly pricing the perishable item. 


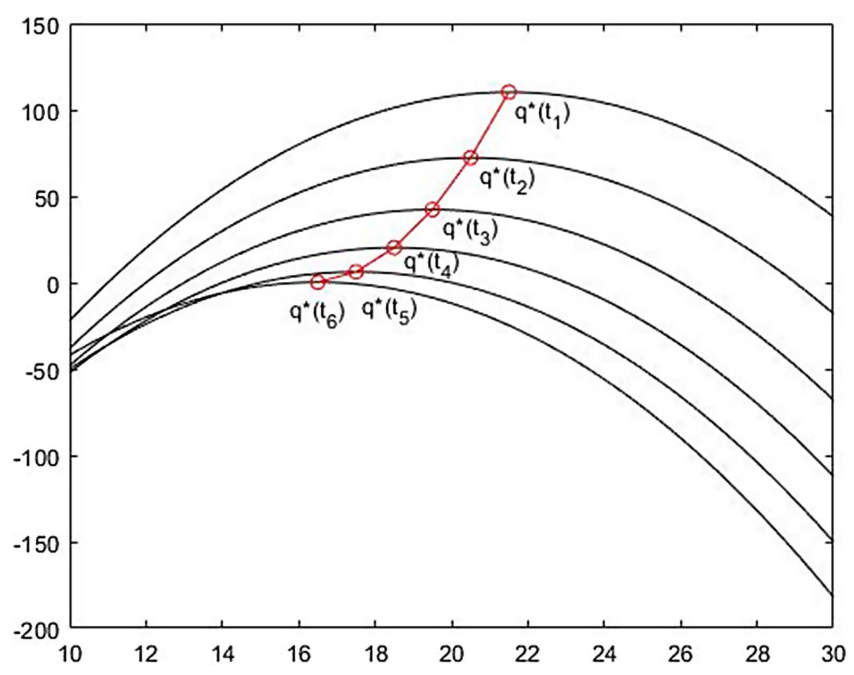

FIGURE 1. Variation trend of $q^{*}\left(t_{i}\right)$.

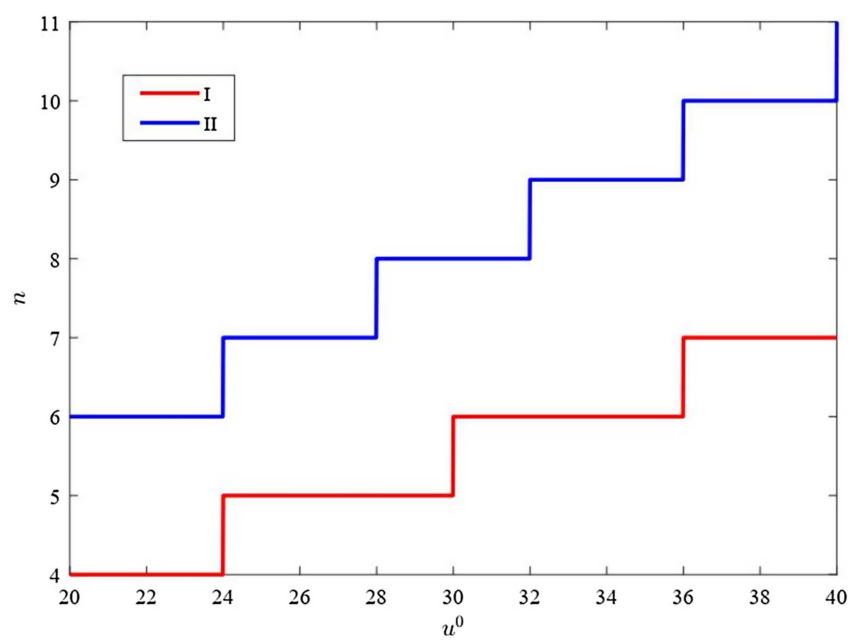

Figure 2. Change curves of the two selling cycle lengths.

Finally, the issue of how the optimal selling cycle length changes under different initial utility values is investigated. Consider $u^{0} \in[20,40]$. Change curves of the two selling cycle lengths for different scenarios are depicted as follows.

In Figure 2, curve I represents the change trajectory of the selling cycle length in the non-cooperation scenario, and curve II represents the change trajectory of the selling cycle length in the cooperation scenario. As it is shown, the selling cycle length is extended by cooperation.

\section{Conclusions}

This paper considers supply and sales issues of perishable items under a price-and time-sensitive demand function. According to whether or not the supplier and the retailer cooperate and jointly price their products, 
we propose two models. In the noncooperative situation, we obtain the optimal strategies for the supplier and the retailer by solving a Stackelberg game model. In the joint dynamic pricing situation, the optimal supply quantity and the optimal sales price are obtained.

Our main theoretical and managerial contributions are summarized as follows:

(1) It is shown that in the cooperative situation, the supplier and the retailer will lower the sales price for the sake of a great raise on the sales quantity.

(2) The optimal selling cycle length of each situation is acquired, which is a study gap till now. And we show that the selling cycle length will be extended by cooperation between the supplier and the retailer.

(3) The increased value incurred by cooperation is proved to be positive, and a method based on the proportion of each previous profit is proposed to allocate the increased profit.

Nevertheless, this paper has some shortcomings. We overlook consumer' strategic behavior. In reality, strategic consumers may arrange their purchasing behavior by considering the retailer's dynamic pricing. Besides, the online channel may need to be taken into consideration. In practice, Wumart supermarket has already operated dual channels to sell perishable products in the presence of inventory sharing. We intend to explore this topic in our next study.

Acknowledgements. We would like to thank the editors and reviewers for their constructive suggestions and corrections to enhance the clarity of the present article.

\section{REFERENCES}

[1] Y. Akcay, H.P. Natarajan and S.H. Xu, Joint dynamic pricing of multiple perishable products under consumer choice. Manage. Sci. 56 (2010) 1345-1361.

[2] D. Besanko and W.L. Winston, Optimal price skimming by a monopolist facing rational consumers. Manage. Sci. 36 (1990) $555-567$.

[3] O. Besbes and D. Saure, Dynamic pricing strategies in the presence of demand shifts. Manuf. Serv. Oper. Manage. 16 (2014) $513-544$.

[4] A.D. Boer and B. Zwart, Dynamic pricing and learning with finite inventories. Oper. Res. 63 (2015) 965-978.

[5] R.E. Chatwin, Optimal dynamic pricing of perishable products with stochastic demand and a finite set of prices. Eur. J. Oper. Res. 125 (2000) 149-174.

[6] Z.X. Chen, Optimization of production inventory with pricing and promotion effort for a single-vendor multi-buyer system of perishable products. Int. J. Prod. Econ. 209 (2018) 285-301.

[7] Y.H. Chen, S.B. Ray and Y.Y. Song, Optimal pricing and inventory control policy in periodic-review systems with fixed ordering cost and lost sales. Nav. Res. Logistics 53 (2006) 117-136.

[8] X. Chen, Z. Pang and L.M. Pan, Coordinating inventory control and pricing strategies for perishable products. Oper. Res. 62 (2014) 284-300.

[9] C.Y. Dye and C.T. Yang, Optimal dynamic pricing and preservation technology investment for deteriorating products with reference price effects. Omega 62 (2016) 52-67.

[10] C.Y. Dye, C.T. Yang and C.C. Wu, Joint dynamic pricing and preservation technology investment for an integrated supply chain with reference price effects. J. Oper. Res. Soc. 69 (2017) 811-824.

[11] L. Feng, Dynamic pricing, quality investment, and replenishment model for perishable items. Int. Trans. Oper. Res. 26 (2019) $1558-1575$.

[12] G. Gallego and M. Hu, Dynamic pricing of perishable assets under competition. Manage. Sci. 60 (2014) 1241-1259.

[13] G. Gallego and G.V. Ryzin, Optimal dynamic pricing of inventories with stochastic demand over finite horizons. Manage. Sci. 40 (1994) 999-1020.

[14] M. Guajardo, M. Kylinger and M. Ronnqvist, Joint optimization of pricing and planning decisions in divergent supply chain. Int. Trans. Oper. Res. 20 (2013) 889-916.

[15] J.X. Jia and Q.Y. Hu, Dynamic ordering and pricing for a perishable goods supply chain. Comput. Ind. Eng. 60 (2011) 302-309.

[16] N.B. Keskin and A. Zeevi, Dynamic pricing with an unknown demand model: asymptotically optimal semi-myopic policies. Oper. Res. 62 (2014) 1142-1167.

[17] Y. Levin, J. McGill and M. Nediak, Dynamic pricing in the presence of strategic consumers and oligopolistic competition. Manage. Sci. 55 (2009) 32-46.

[18] Y. Levin, J. McGill and M. Nediak, Optimal dynamic pricing of perishable items by a monopolist facing strategic consumers. Prod. Oper. Manage. 19 (2010) 40-60. 
[19] S.K. Li, J.X. Zhang and W.S. Tang, Joint dynamic pricing and inventory control policy for a stochastic inventory system with perishable products. Int. J. Prod. Res. 53 (2015) 2937-2950.

[20] Q. Liu and D. Zhang, Dynamic pricing competition with strategic customers under vertical product differentiate. Manage. Sci. 59 (2013) 84-101.

[21] G.W. Liu, J.X. Zhang and W.S. Tang, Joint dynamic pricing and investment strategy for perishable foods with price-quality dependent demand. Ann. Oper. Res. 226 (2015) 397-416.

[22] Z.K. Lou, F.J. Hou and X.M. Lou, Optimal ordering and pricing models of a two-echelon supply chain under multiple times ordering. J. Ind. Manage. Optim. (2020) 1-13.

[23] J. Lu, J.X. Zhang and X.Y. Jia, Optimal dynamic pricing, preservation technology investment and periodic ordering policies for agricultural products. RAIRO: OR $\mathbf{5 3}$ (2019) 731-747.

[24] V. Mak, A. Rapoport and E.J. Gisches, Competitive dynamic pricing with alternating offers: theory and experiment. Games Econ. Behav. 75 (2012) 250-264.

[25] Y. Papanastasiou and N. Savva, Dynamic pricing in the presence of social learning and strategic consumers. Manage. Sci. 63 (2017) 919-939.

[26] S. Saha, S. Majumder and I.E. Nielsen, Is it a strategic move to subsidized consumers instead of the manufacturer? IEEE Access 7 (2019) 169807-169824.

[27] R. Schlosser and M. Boissier, Dealing with the dimensionality curse in dynamic pricing competition: using frequent repricing to compensate imperfect market anticipations. Comput. Oper. Res. 100 (2018) 26-42.

[28] A.A. Taleizadeh and N.D. Mahsa, Pricing, manufacturing and inventory policies for raw material in a three-level supply chain. Int. J. Syst. Sci. 47 (2016) 919-931.

[29] R. Weatherford and S. Bodily, A taxonomy and research overview of perishable asset revenue management: yield management, overbooking and pricing. Oper. Res. 40 (1992) 831-844.

[30] F. Zabihi and M.K. Bafruei, Pricing and determining the optimal discount time of perishable goods with time and price dependent demand. RAIRO: OR $\mathbf{5 1}$ (2017) 509-518. 